Supporting Information

\title{
Feather-like NiCoP Holey Nanoarrys for Efficient and Stable Seawater Splitting
}

Qingliang Lv, ${ }^{\dagger}$ Jianxin Han, ${ }^{\dagger}$ Xueling Tan, ${ }^{\dagger}$ Wei Wang, ${ }^{\dagger}, \dagger$ Lixin Cao, $*, \dagger$ and Bohua Dong $*, \dagger$

${ }^{\dagger}$ School of Materials Science and Engineering, Ocean University of China, 238

Songling Road, Qingdao, 266100 P. R. China

${ }^{\ddagger}$ Aramco Research Center-Boston, Aramco Services Company, Cambridge, MA 02139, USA

Corresponding author: dongbohua@ouc.edu.cn; caolixin@ouc.edu.cn 


\section{Contents:}

Figure S1 SEM image of the $\mathrm{Ni}_{2} \mathrm{P}$ and $\mathrm{CoP}$.

Figure S2 XRD pattern of the as-prepared sample.

Figure S3 XRD pattern of NiCo-precursor.

Figure S4 HER polarization curves obtained with the PF-NiCoP/NF electrode in $1 \mathrm{M}$ $\mathrm{KOH}$ solution and seawater without correcting the iR drop.

Figure S5 CVs of (a) the NiCoP/NF, (b) $\mathrm{Ni}_{2} \mathrm{P} / \mathrm{NF}$, (c) CoP/Ti, (d) bare Ni Foam with various scan rates $\left(20-200 \mathrm{mV} \mathrm{s}^{-1}\right)$.

Figure S6 polarization curves related electrocatalysts for OER and overall water splitting with $90 \%$ iR compensation recorded at a scan rate of $1 \mathrm{mV} \mathrm{s}^{-1}$ in $1 \mathrm{M} \mathrm{KOH}$.

Figure S7 XPS of Ni $2 p_{3 / 2}$, Co $2 p_{3 / 2}$ and P $2 p$ spectrum in NiCoP before and after OER tests.

Figure S8 SEM of the porous feather-like NiCoP/NF after OER tests.

Figure S9 EDS of the porous feather-like $\mathrm{NiCoP} / \mathrm{NF}$ after OER tests.

Table S1 A comparative summary of HER activities. 


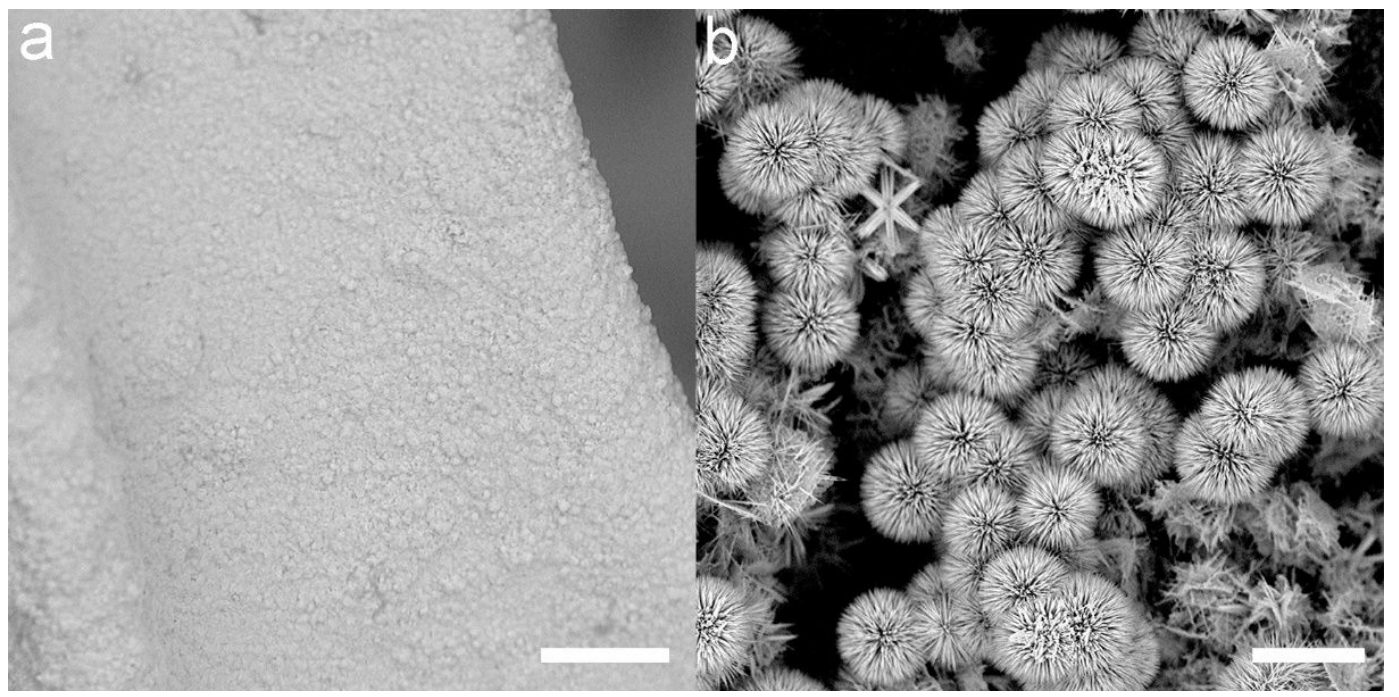

Figure S1. SEM image of the $\mathrm{Ni}_{2} \mathrm{P}$ and $\mathrm{CoP} . \mathrm{Ni}_{2} \mathrm{P}$ nanoparticles (Figure $\mathrm{S} 1 \mathrm{a}$ )

uniformly grown on $\mathrm{Ni}$ foam after $\mathrm{Ni}$ foam phosphating. Moreover, $\mathrm{CoP}$ nanoflowers

(Figure S1b) obtained after phosphating are uniformly grown on Ti sheets. The scale bar of $\mathrm{a}$ and $\mathrm{b}$ are $10 \mu \mathrm{m}$ and $20 \mu \mathrm{m}$, respectively. 


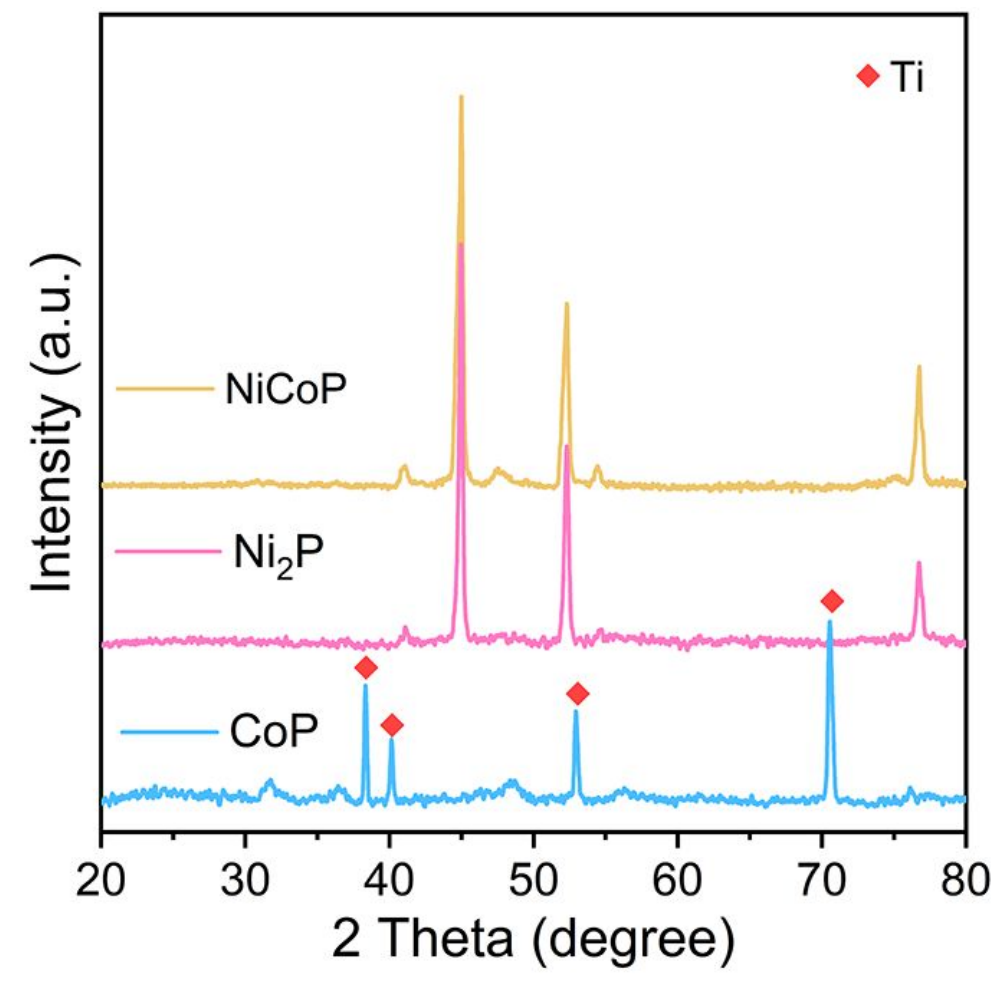

Figure S2. XRD pattern of the as-prepared sample. 


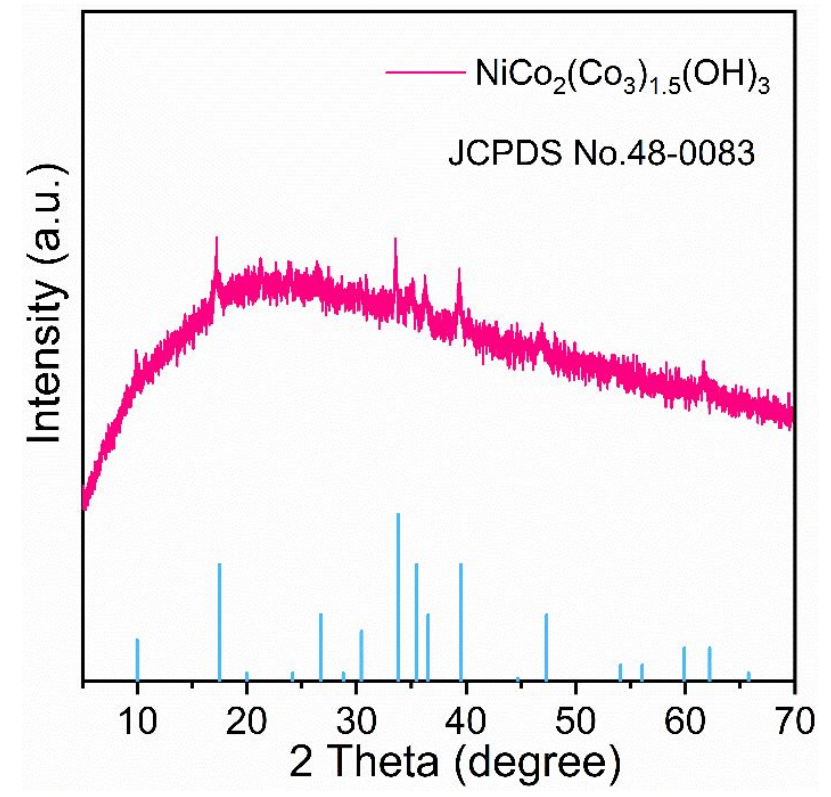

Figure S3. XRD pattern of NiCo-precursor. The diffraction data is readily indexed to $\mathrm{NiCo}_{2}\left(\mathrm{Co}_{3}\right)_{1.5}(\mathrm{OH})_{3}(\mathrm{JCPDS}$ Card No. 48-0083), without discernible diffraction peaks of other phases. 

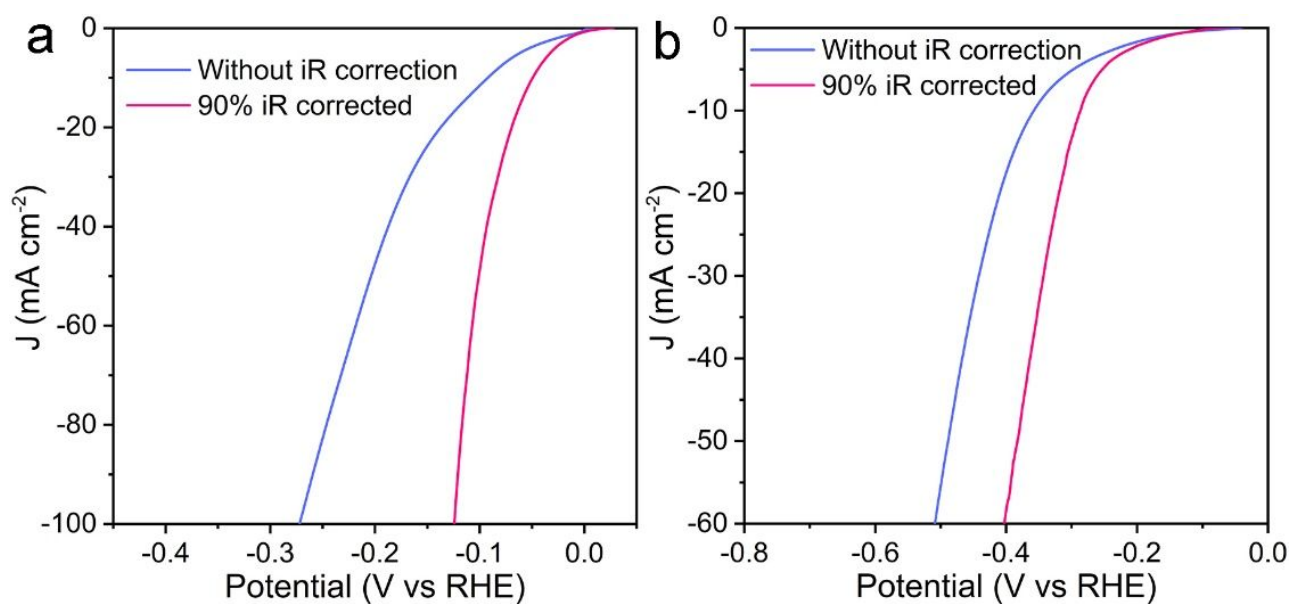

Figure S4. HER polarization curves obtained with the PF-NiCoP/NF electrode in $1 \mathrm{M}$ $\mathrm{KOH}$ solution and seawater with and without $\mathrm{R}$ compensation. The scan rate is $1 \mathrm{mV}$ $\mathrm{s}^{-1}$. 

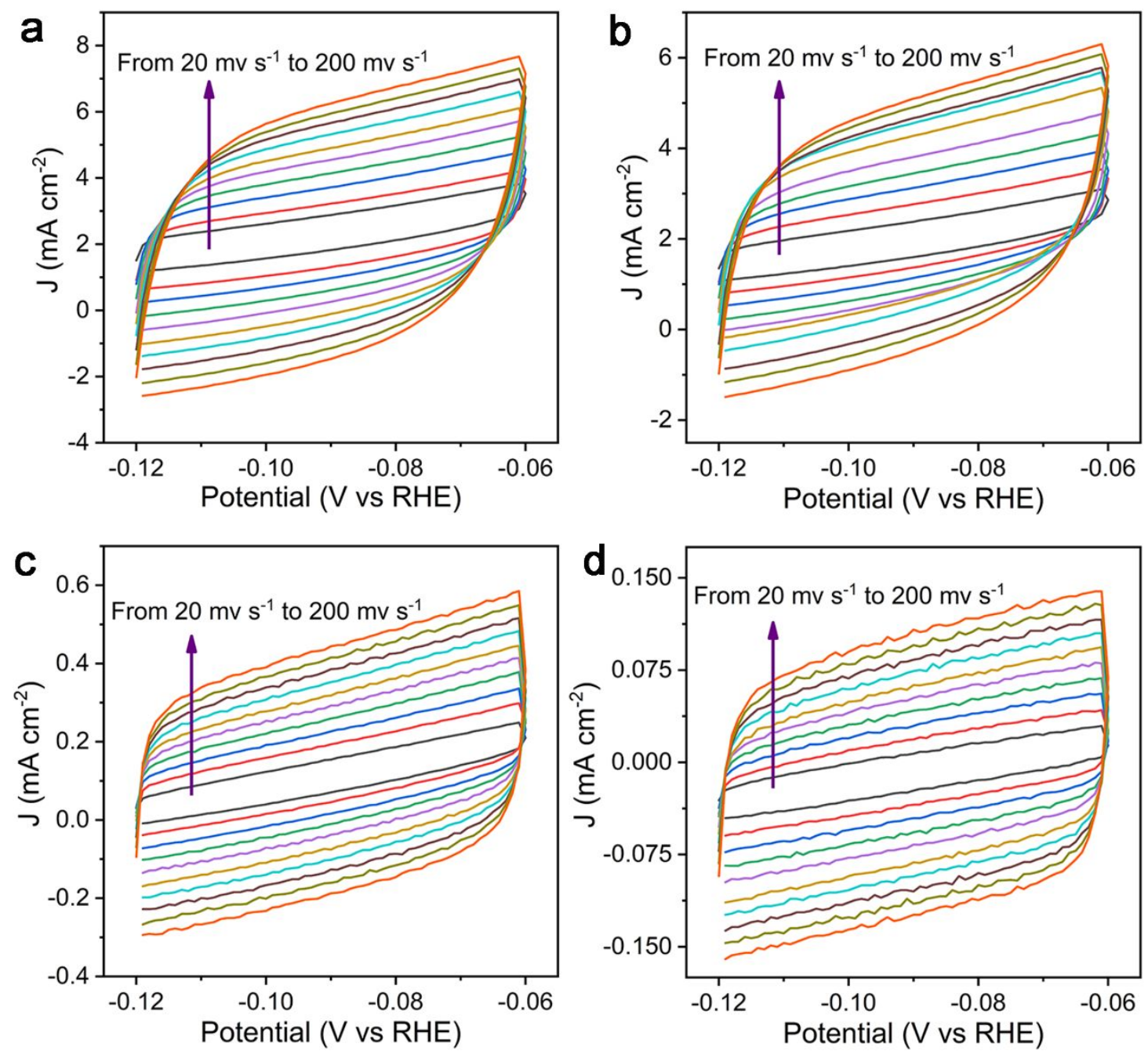

Figure S5. CVs of (a) the $\mathrm{NiCoP} / \mathrm{NF}$, (b) $\mathrm{Ni}_{2} \mathrm{P} / \mathrm{NF}$, (c) $\mathrm{CoP} / \mathrm{Ti}$, (d) bare Ni Foam with various scan rates $\left(20-200 \mathrm{mV} \mathrm{s}^{-1}\right)$. 

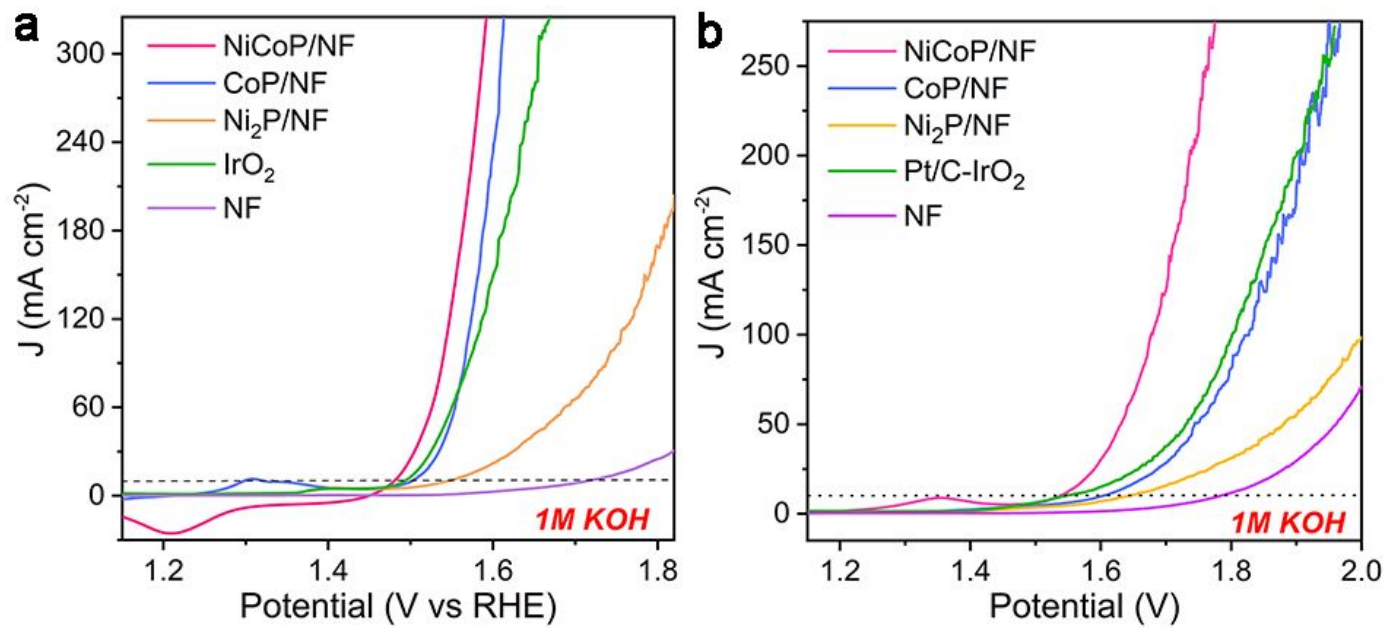

Figure S6. (a) polarization curves per geometric area of related electrocatalysts for OER with $90 \%$ iR compensation recorded at a scan rate of $1 \mathrm{mV} \mathrm{s}^{-1}$ in $1 \mathrm{M} \mathrm{KOH}$. (b) LSV curves of the related electrocatalysts for overall water splitting with $90 \% \mathrm{iR}$ compensation recorded at a scan rate of $1 \mathrm{mV} \mathrm{s}^{-1}$ in $1 \mathrm{M} \mathrm{KOH}$. 

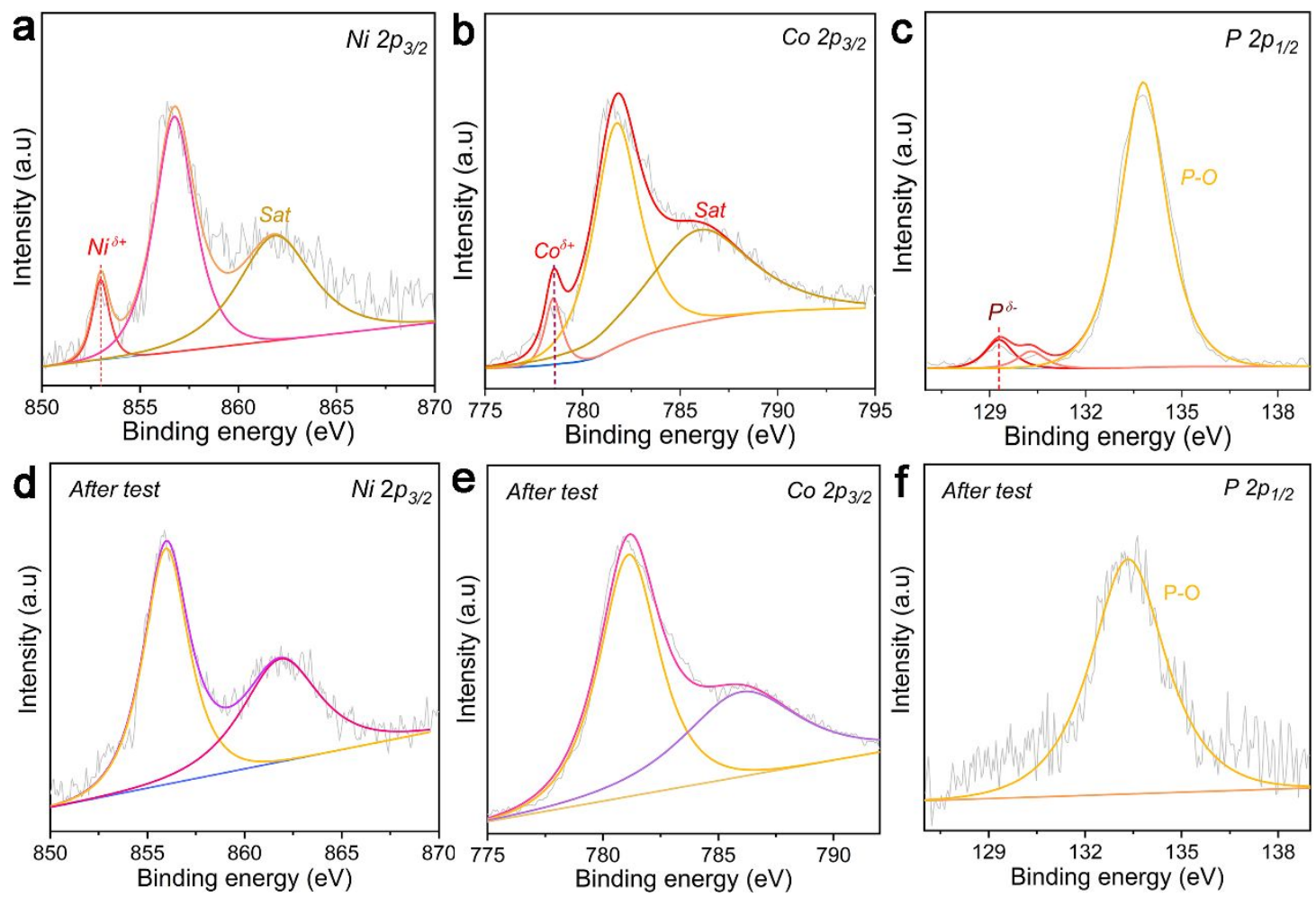

Figure S7. XPS of Ni $2 p_{3 / 2}$, Co $2 p_{3 / 2}$ and $\mathrm{P} 2 \mathrm{p}$ spectrum in NiCoP before and after OER tests 


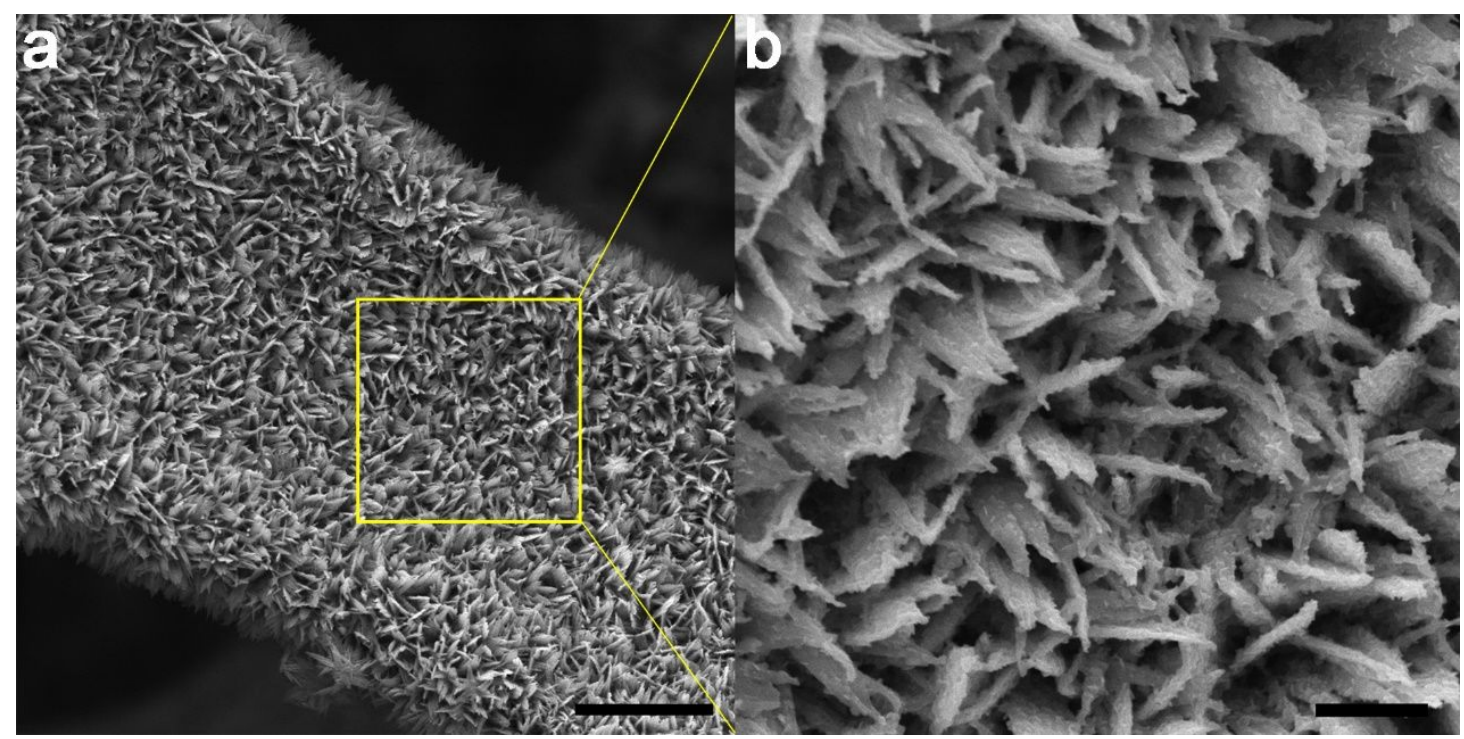

Figure S8. SEM of the porous feather-like NiCoP/NF after OER tests. The scale bar of $\mathrm{a}$ and $\mathrm{b}$ are $20 \mu \mathrm{m}$ and $5 \mu \mathrm{m}$, respectively. 


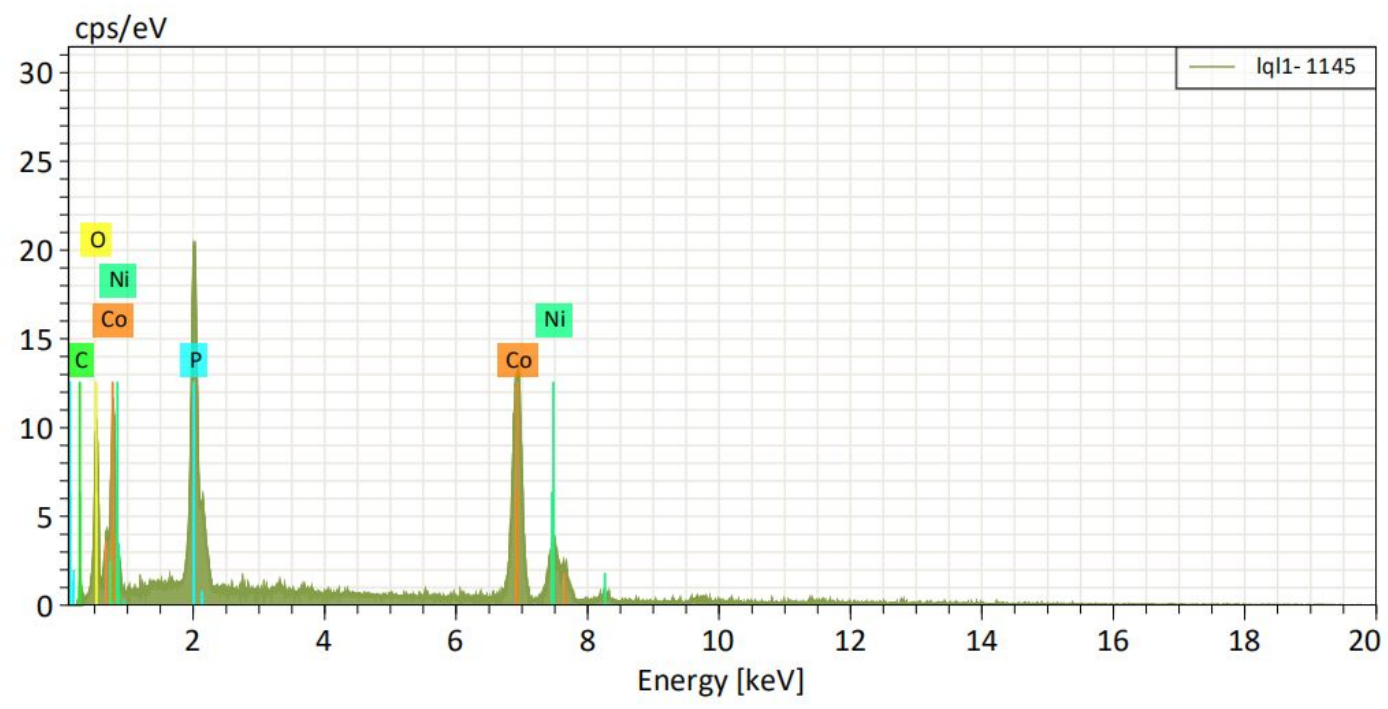

Figure S9. EDS of the porous feather-like NiCoP/NF after OER tests 
Table S1. Comparison of HER performance for porous feather-like NiCoP/NF with other nonnoble-metal HER electrocatalysts in alkaline solution

\begin{tabular}{|c|c|c|c|c|}
\hline Electrocatalyst & Electrolyte & $\underset{\mathbf{c m}^{-2}}{\mathbf{1} @ \mathbf{1 0 m} A}$ & $\begin{array}{l}\text { Tafel slop } \\
\left(\mathrm{mV} \mathrm{dec}^{-1}\right)\end{array}$ & Reference \\
\hline Porous feather-like NiCoP & $1 \mathrm{M} \mathrm{KOH}$ & 46 & 54 & This work \\
\hline NiCoP@Ru & $1 \mathrm{M} \mathrm{KOH}$ & 52 & 50 & 1 \\
\hline Whisker-on-sheet NiCoP & $1 \mathrm{M} \mathrm{KOH}$ & 59 & - & 2 \\
\hline NiCoP holey nanosheets & $1 \mathrm{M} \mathrm{KOH}$ & 58 & 57 & 3 \\
\hline Nest-like NiCoP & $1 \mathrm{M} \mathrm{KOH}$ & 62 & 39 & 4 \\
\hline Ternary NiCoP nanosheet & $1 \mathrm{M} \mathrm{KOH}$ & 100 & 68.6 & 5 \\
\hline Multishelled $\mathrm{Ni}_{2} \mathrm{P}$ & $1 \mathrm{M} \mathrm{KOH}$ & 98 & 86.4 & 6 \\
\hline $\mathrm{Co}_{2} \mathrm{P} @ \mathrm{CP}$ & $1 \mathrm{M} \mathrm{KOH}$ & 70 & 59.7 & 7 \\
\hline $\mathrm{FeP} \mathrm{NAs} / \mathrm{CC}$ & $1 \mathrm{M} \mathrm{KOH}$ & 218 & 146 & 8 \\
\hline $\mathrm{Ni}_{2} \mathrm{P}$ & $1 \mathrm{M} \mathrm{KOH}$ & 176 & - & 9 \\
\hline NiCoP hollow polyhedra & $1 \mathrm{M} \mathrm{KOH}$ & 142 & 42 & 10 \\
\hline $\mathrm{No}_{0.51} \mathrm{Co}_{0.49} \mathrm{P}$ & $1 \mathrm{M} \mathrm{KOH}$ & 82 & 43 & 11 \\
\hline $\mathrm{Co}_{2} \mathrm{P}$ nanorods & $1 \mathrm{M} \mathrm{KOH}$ & 134 & 71 & 12 \\
\hline $\mathrm{NiSe}$ & $1 \mathrm{M} \mathrm{KOH}$ & 96 & 120 & 13 \\
\hline $\mathrm{NiCo}_{2} \mathrm{P}_{\mathrm{x}} / \mathrm{CF}$ & $1 \mathrm{M} \mathrm{KOH}$ & 58 & 34.3 & 14 \\
\hline CoMoP@C & $1 \mathrm{M} \mathrm{KOH}$ & 81 & 55.5 & 15 \\
\hline 3D-NiCoP & $1 \mathrm{M} \mathrm{KOH}$ & 105 & 79 & 16 \\
\hline $\mathrm{CoP} / \mathrm{Ni}_{5} \mathrm{P}_{4} / \mathrm{CoP}$ & $1 \mathrm{M} \mathrm{KOH}$ & 71 & 43 & 17 \\
\hline NiCoP nanocone arrays/NF & $1 \mathrm{M} \mathrm{KOH}$ & 104 & 54 & 18 \\
\hline $\mathrm{Ni}_{0.33} \mathrm{Co}_{0.67} \mathrm{Se}_{2}$ & $1 \mathrm{M} \mathrm{KOH}$ & 106 & 60 & 19 \\
\hline $\mathrm{Ni}_{0.89} \mathrm{Co}_{0.11} \mathrm{Se}_{2} \mathrm{MNSN} / \mathrm{NF}$ & $1 \mathrm{M} \mathrm{KOH}$ & 85 & 52 & 20 \\
\hline
\end{tabular}




\section{Reference:}

1. Liu, S.; Liu, Q.; Lv, Y.; Chen, B. Y.; Zhou, Q.; Wang, L.; Zheng, Q. H.; Che, C. J.; Chen, C. Y. Ru Decorated with NiCoP: An Efficient and Durable Hydrogen Evolution Reaction Electrocatalyst in both Acidic and Alkaline Conditions. Chem. Commun. 2017, 53, 1315313156.

2. Cai, Z.; Wu, A.; Yan, H.; Xiao, Y.; Chen, C.; Tian, C.; Wang, L.; Wang, R.; Fu, H. Hierarchical Whisker-on-Sheet NiCoP with Adjustable Surface Structure for Efficient Hydrogen Evolution Reaction. Nanoscale 2018, 10, 7619-7629.

3. Fang, Z.; Peng, L.; Qian, Y.; Zhang, X.; Xie, Y.; Cha, J. J.; Yu, G. H. Dual Tuning of Ni-Co$\mathrm{A}(\mathrm{A}=\mathrm{P}, \mathrm{Se}, \mathrm{O})$ Nanosheets by Anion Substitution and Holey Engineering for Efficient Hydrogen Evolution. J. Am. Chem. Soc. 2018, 140, 5241-5247.

4. Du, C.; Yang, L.; Yang, F.; Cheng, G. Z.; Luo, W. Nest-like NiCoP for Highly Efficient Overall Water Splitting. ACS Catal. 2017, 7, 4131-4137.

5. Li, Y.; Zhang, H.; Jiang, M.; Kuang, Y.; Sun, X.; Duan, X. Ternary NiCoP Nanosheet Arrays: An Excellent Bifunctional Catalyst for Alkaline Overall Water Splitting. Nano Res. 2016, 9, 2251-2259.

6. Sun, H. M.; Xu, X. B.; Yan, Z. H.; Chen, X.; Cheng, F. Y.; Weiss, P. S.; Chen, J. Porous Multishelled $\mathrm{Ni}_{2} \mathrm{P}$ Hollow Microspheres as an Active Electrocatalyst for Hydrogen and Oxygen Evolution. Chem. Mater. 2017, 29, 8539-8547.

7. Zhang, Y.; Gao, L.; Hensen, E. J. M.; Hofmann, J. P. Evaluating the Stability of $\mathrm{Co}_{2} \mathrm{P}$ Electrocatalysts in the Hydrogen Evolution Reaction for Both Acidic and Alkaline Electrolytes. ACS Energy Lett. 2018, 3, 1360-1365.

8. Liang, Y.; Liu, Q.; Asiri, A. M.; Sun, X.; Luo, Y. Self-supported FeP Nanorod Arrays: A Cost-Effective 3D Hydrogen Evolution Cathode with High Catalytic Activity. ACS Catal. 2014, 4, 4065-4069.

9. Menezes, P. W., Indra, A., Das, C., Walter, C., Göbel, C., Gutkin, V., Schmeißer, D.; Driess, M. Uncovering the Nature of Active Species of Nickel Phosphide Catalysts in HighPerformance Electrochemical Overall Water Splitting. ACS Catal. 2017, 7, 103-109.

10. Li, Y. P.; Liu, J. D.; Chen, C.; Zhang, X. H.; Chen, J. H. Preparation of NiCoP Hollow QuasiPolyhedra and Their Electrocatalytic Properties for Hydrogen Evolution in Alkaline Solution. ACS Appl. Mater. Interfaces 2017, 9, 5982-5991.

11. Wang, X. G.; Li, W.; Xiong, D. H.; Petrovykh, D. Y.; Liu, L. F. Bifunctional Nî̉ckel Phosphide Nanocatalysts Supported on Carbon Fiber Paper for Highly Efficient and Stable Overall Water Splitting. Adv. Funct. Mater. 2016, 26, 4067-4077.

12. Huang, Z. P.; Chen, Z. Z.; Chen, Z. B.; Lv, C. C.; Humphrey, M. G.; Zhang, C. Cobalt Phosphide Nanorods as An Efficient Electrocatalyst for the Hydrogen Evolution Reaction. Nano Energy, 2014, 9, 373-382. 
13. Tang, C.; Cheng, N. Y.; Pu, Z. H.; Xing, W.; Sun, X. P. NiSe Nanowire Film Supported on Nickel Foam: An Efficient and Stable 3D Bifunctional Electrode for Full Water Splitting. Angew. Chem. Int. Ed. 2015, 54, 9483- 9487.

14. Zhang, R.; Wang, X.; Yu, S.; Wen, T.; Hu, W. Ternary $\mathrm{NiCo}_{2} \mathrm{P}_{\mathrm{x}}$ Nanowires as pH-Universal Electrocatalysts for Highly Efficient Hydrogen Evolution Reaction. Adv. Mater. 2017, 29, 1605502.

15. Ma, Y. Y.; Wu, C.X.; Feng, X. J.; Tan, H. Q.; Yan, L. K.; Liu, Y.; Kang, Z. H.; Wang, E. B.; Li, Y.G. Highly Efficient Hydrogen Evolution from Seawater by a Low-Cost and Stable CoMoP@C Electrocatalyst Superior to Pt/C. Energy Environ. Sci. 2017, 10, 788-798.

16. Ma, B.; Yang, Z.; Chen, Y.; \& Yuan, Z. Nickel Cobalt Phosphide with Three-Dimensional Nanostructure as a Highly Efficient Electrocatalyst for Hydrogen Evolution Reaction in both Acidic and Alkaline Electrolytes. Nano Res. 2019, 12, 375-380.

17. Mishra, I. K.; Zhou, H.; Sun, J.; Qin, F.; Dahal, K.; Bao, J.; Chen, S.; Ren, Z. F. Hierarchical $\mathrm{CoP} / \mathrm{Ni}_{5} \mathrm{P}_{4} / \mathrm{CoP}$ Microsheet Arrays as a Robust pH-Universal Electrocatalyst for Efficient Hydrogen Generation. Energy Environ. Sci. 2018, 11, 2246-2252.

18. Li, J.; Wei, G.; Zhu, Y.; Xi, Y.; Pan, X.; Ji, Y. Hierarchical NiCoP Nanocone Arrays Supported on Ni Foam as An Efficient and Stable Bifunctional Electrocatalyst for Overall Water Splitting. J. Mater. Chem. A 2017, 5, 14828-14837.

19. Xia, C.; Liang, H.; Zhu, J.; Schwingenschlögl, U.; Alshareef, H. N. Active Edge Sites Engineering in Nickel Cobalt Selenide Solid Solutions for Highly Efficient Hydrogen Evolution. Adv. Energy Mater. 2017, 7, 1602089.

20. Liu, B.; Zhao, Y. F.; Peng, H. Q.; Zhang, Z. Y.; Sit, C. K.; Yuen, M. F.; Zhang, T. R.; Lee, C. S.; Zhang, W. J. Nickel-Cobalt Diselenide 3D Mesoporous Nanosheet Networks Suppofted on Ni Foam: An All-pH Highly Efficient Integrated Electrocatalyst for Hydrogen Evolution. ^Adv. Mater. 2017, 29, 1606521. 